\title{
A Computer-Controlled Submillimeter Fourier Spectrometer
}

\author{
JAGDISH K. VIJ AND PATRICK CORCORAN
}

\begin{abstract}
A system for a submillimeter wave Fourier transform spectroscopy (FTS) has been automated for the measurement of the power absorption coefficient of liquids in the wavenumber range 10$400 \mathrm{~cm}^{-1}$. The interferometer is interfaced with a RM380Z Research Machines microcomputer. Software and hardware have been developed to automate the process of collecting and storing the data. Some points of relevance to the analysis of interferometric data are reviewed including the use of phase modulation, the resolution of spectra computed using the discrete Fourier transform, and the necessity of proper optical filtering in spectrometers which use Fourier transform techniques. Spectra measured using the FTS system described here have been compared with results obtained recently using a tunable submillimeter laser and a good agreement is found between the two sets of data.
\end{abstract}

\section{INTRODUCTION}

$\mathrm{D}$ URING THE 1950's Gebbie, Vanasse, and Strong developed a Fourier transform spectrometer suitable for measurements in the wavenumber range $0-100 \mathrm{~cm}^{-1}$ [1], [2]. The spectrometer utilizes the Michelson interferometer whereby the interference of electromagnetic radiation from an incoherent broad-band source is produced by superposition of two wave trains obtained by a beam splitter. The optical parts of the spectrometer are in modular form and can be assembled to any convenient geometrical configuration. The electronic system initially available consisted of electrical switches and relays with the sampled interferogram being produced on a punched tape. This was later fed to a mainframe computer which carried out the Fourier transformation and the other computation required to produce spectra. The spectrometer was manufactured by Grubb-Parsons Ltd. based on a design from the N.P.L., U.K. and was used unaltered in many research laboratories until the early 1980's. We have interfaced the interferometer with an RM380Z Research Machines microcomputer (RMC). The microcomputer now controls the spectrometer and stores the digitally recorded interferogram on a floppy disk. The sampled interferogram is then passed on to a microcomputer where the Fourier transformation and other computation is carried out to produce on-line transmission and absorption spectra of the material under investigation.

Manuscript received September 25, 1987; revised September 19, 1988 This work was supported in part by Trinity Trust T.C.D., Dublin, Ireland The authors are with the Department of Microelectronics and Electrical Engineering, Trinity College Dublin, Dublin 2, Ireland.

IEEE Log Number 8824659.

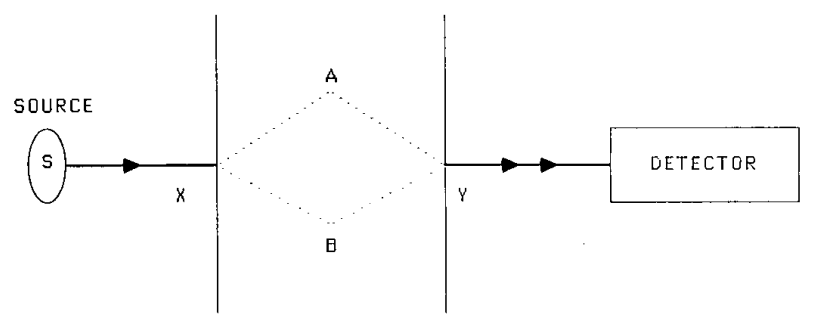

Fig. 1. Schematic diagram of a two-beam interferometer.

\section{Principles of the Optical System}

With reference to Fig. 1, the partial beam of light travels along a path greater than that traversed by the beam $X B Y$ by a distance of $x(0 \leq x \leq \infty)$ where $x / 2$ is the difference in the distance of two mirrors from the beam divider. We initially assume monochromatic radiation of wavelength $\lambda$. The optical path difference between the two beams gives rise to a relative phase difference of $2 \pi x / \lambda$ radians or $2 \pi \bar{\nu} x$, where $\bar{\nu}=1 / \lambda$. If the source power lies in the range $\bar{\nu}$ to $\bar{\nu}+d \bar{\nu}$, the power at the detector $I(x)$ is

$$
I(x)=B(\bar{\nu}) d \bar{\nu}+B(\bar{\nu}) \cos (2 \pi \bar{\nu} x) d \bar{\nu}
$$

assuming that the two partial beams are equal in intensity and each has a spectral intensity $\frac{1}{2} B(\bar{\nu}) d \bar{\nu}$ in the previously defined range [3]. A broad-band source produces incoherent radiation composed of a chaotic assembly of wave trains of random length and phase. Interference is produced by the recombination of the individual wavelets after reflection from the surfaces of the interferometer mirrors. The detected power is

$$
I(x)=\int_{0}^{\infty} B(\bar{\nu}) d \bar{\nu}+\int_{0}^{\infty} B(\bar{\nu}) \cos (2 \pi \bar{\nu} x) d \bar{\nu}
$$

Let

$$
b(x) \equiv I(x)-\frac{1}{2} I(0)=\int_{0}^{\infty} B(\bar{\nu}) \cos (2 \pi \bar{\nu} x) d \bar{\nu}
$$

making use of the fact that at $x=0$ all the components are in phase and consequently $I(0)=2 \int_{0}^{\infty} B(\bar{\nu}) d \bar{\nu}$ from (2). The function $b(x)$ is called the interferogram and is the variable part of the detected interference function. $I(0)$ is the steady "background" or average value. The function $B(\bar{\nu})$ is the Fourier cosine transform and thus 


$$
B(\bar{\nu})=\int_{0}^{\infty} b(x) \cos (2 \pi \bar{\nu} x) d x .
$$

Although $B(\vec{\nu})$ is not directly accessible it may be derived by Fourier transformation of $b(x)$ which is a measurable quantity.

The above treatment deals with unmodulated radiation. In practice it is usual to modulate the light beam from the source to improve the signal-to-noise ratio of the detected signal. Modulation is also necessary if the detector is a Golay thermal detector which does not respond to steady, unmodulated radiation. Amplitude modulation (AM) is achieved by means of a rotating chopper which periodically interrupts the beam from the source. An AM interferogram is symmetrical about the zero path-difference position (ZPD) and a Fourier cosine transform is still appropriate for deriving the required spectral information. An alternative method of modulation is phase modulation (PM) [4] where the fixed mirror of the interferometer is "jittered" back and forth about a mean position with its surface kept perpendicular to the axis. In the case of PM the signal measured is that which depends on the phase difference $2 \pi \bar{\nu} x$. The average dc signal and electrical noise are not included leading to a considerable enhancement of the signal-to-noise ratio of the system. Since there is no "chopping" of the radiation, the PM technique makes full use of the power available from the source. A phase modulated interferogram is antisymmetric about the ZPD position. It can be shown [4] that if the modulating signal is cosinusoidal

$$
b(x) \propto \int_{0}^{\infty} B(\bar{\nu}) J_{1}(2 \pi \bar{\nu} a) \sin (2 \pi \bar{\nu} x) d \bar{\nu}
$$

where $J_{1}$ is a Bessel function of the first order and $a$ is the amplitude of the modulation in terms of optical path difference. In this case, a Fourier sine transform is required to recover spectral information from the interferogram.

\section{SYSTEM HARDWARE}

A block diagram of the system is shown in Fig. 2. The source in the cube used is a Philips medium pressure mercury lamp, type HPK $125 \mathrm{~W}$, which generates broad-band submillimeter radiation by thermal emission from its quartz envelope. The fixed mirror of the interferometer (M2) is mounted on a diaphragm which may be connected to an oscillator to phase modulate the detected signal. The moveable mirror $(M 1)$ is mounted on a micrometer which is driven by a stepper motor. A thin stretched dielectric film (polyethylene or melinex) is used as the beam divider (BD). The detector is a low-noise Golay infrared detector, type IR50. The detector is fitted with a diamond window which allows measurements to be made up to about $400 \mathrm{~cm}^{-1}$. The output from the detector is fed to a phase sensitive amplifier (ORTEC-Brookdeal 9501). This is a lock-in amplifier [5] with a broad-band frequency range of $0.5 \mathrm{~Hz}-150 \mathrm{kHz}$ and a resolution of 10 $\mu \mathrm{V}$. The stepper motor, Evershed hybrid stepping motor (type FDS/4/A51), which drives the movable mirror of

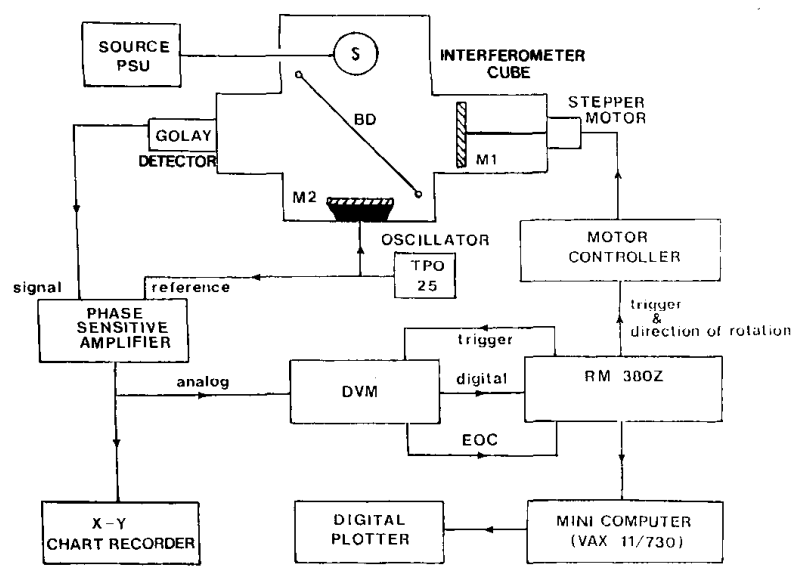

Fig. 2. Block diagram of automated interferometric spectrometer system.

the interferometer is connected to the RMC parallel input/ output (PIO) board via a Digiplan SD2 bipolar driver circuit.

It was found that the recommended $24-\mathrm{V}$ supply voltage across the motor windings created strong magnetic fields which caused electromagnetic induction in the coil driving the diaphragm on which the fixed mirror, $M 2$, is mounted. Due to this, spurious signals were generated on the reference input to the lock-in amplifier. This interfered with the functioning of the amplifier which requires a "clean," unambiguous reference signal for proper lockin detection. The problem was overcome by reducing the motor supply voltage to $4 \mathrm{~V}$, thus reducing the magnetic fields generated in the motor windings. To step the motor an input pulse is applied to the driver unit. Stepping occurs on the rising edge of a pulse which has been low for at least $10 \mu \mathrm{s}$. One complete revolution of the motor requires 200 steps and this corresponds to a mirror travel of $0.5 \mathrm{~mm}$. Thus one motor step produces a mirror displacement of $2.5 \mu \mathrm{m}$.

The digital voltmeter (DVM) is constructed around an RS7138 [6], which is an analog-to-digital (A/D) conversion chip operating on the principal of dual-slope conversion [5]. It provides a 4 digit binary-coded decimal (BCD) output. The RS7138 is clocked at $160 \mathrm{kHz}$ and the clocking signal is derived from a CMOS 7555 timer chip [6]. An A/D conversion is initiated by a high signal of duration greater than 300 ns on the "start conversion" (SC) input of the unit. The conversion time is $250 \mathrm{~ms}$ and upon completion the 3 least significant bytes are latched using TTL 744175 quad latches, where they can be accessed by the RMC. The DVM has an "end-of-convert"' (EOC) output which is low when the unit is idle. During the conversion, the EOC line goes high and returns to the low state when the conversion is complete. During the recording of an interferogram the $\mathrm{RMC}$ monitors this line to ensure that a conversion has been completed before data is read in from the quad latches.

The RMC mini-disk system is used to automate the collection of the interferometric data. The disk controller 


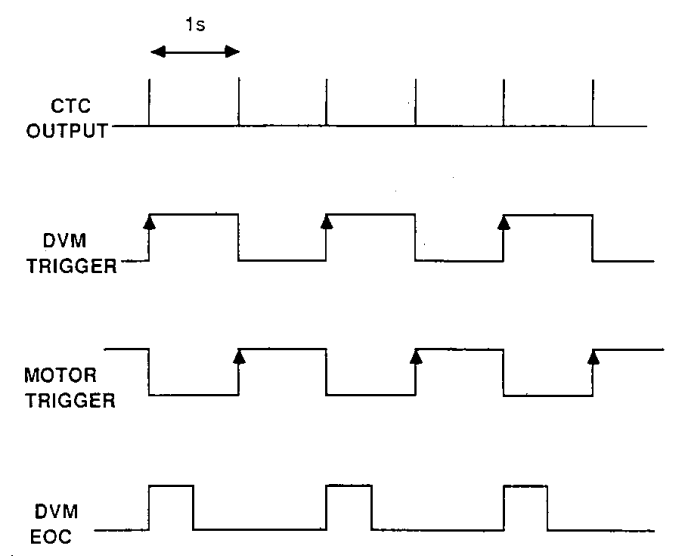

Fig. 3. Timing diagrams showing trigger signals to DVM and stepper motor. Arrows on waveforms indicate edges on which triggering occurs.

board also features a serial interface port which can drive an RS232 interface. We used this to transfer the data collected by the RMC to a VAX $11 / 730$ minicomputer for processing. A separate PIO board allows the transfer of data between the RMC and any peripheral device connected to it-in this case the stepper motor and the DVM. Two Z80 PIO chips are used in our system-one for the output and monitoring of control signals and the other for reading in digitized data from the DVM.

A Z80 counter/timer circuit (CTC) chip is used to provide a continuous train of pulses for triggering the stepper motor and the DVM. The pulsewidth which is controlled by the software is approximately $250 \mathrm{~ns}$ for a system driven by a 4-MHz clock. Such pulses are too short to trigger either the motor driver unit or the DVM. The pulse train is modified by connecting it to the clock input of a $J K$-flip-flop wired up in "toggle" mode $(J=K=$ "' 1 ") . The CTC is programmed to emit pulses at the rate of one per second, giving rise to a $0.5-\mathrm{Hz}$ square wave at the flip-flop output. The DVM and stepper motor are triggered in antiphase (see timing diagram Fig. 3) using the $Q$ and $\bar{Q}$ outputs of the flip-flop. Assuming that the time required to step the motor is negligible, the Golay detector has $1 \mathrm{~s}$ to stabilize before the DVM is triggered and data capture takes place. This arrangement appears quite adequate, since there was no discernible improvement in the results obtained with the CTC programmed to allow the Golay $2 \mathrm{~s}$ to stabilize. An alternative triggering arrangement using a sample-and-hold circuit (SHC) to capture data from the DVM and a monostable delay circuit to subsequently step the motor allowed data to be captured at a somewhat faster rate. However, the introduction of the SHC increased the level of noise present in the digitized data. When no SHC is used the integrating action of the dual-slope converter [5] tends to average out any noise present at the DVM input.

\section{System Control Software}

The program sets up the PIO's and the CTC by writing control words to the relevant registers. It also establishes a link with the VAX via the serial interface port and prompts the user to create a VAX file where the data can be stored. It then enables the CTC to start triggering the DVM and the motor, and then proceeds to record the required interferometric data. A similar program has been developed which allows local storage of data on one of the RMC discs. The data may be transferred to the VAX for processing at a later stage.

\section{Processing the Interferometric Data \\ A. Use of the Complex Fourier Transform}

Where an interferogram is perfectly symmetrical or antisymmetrical about the ZPD position it is, in principle, possible to record the interferogram over a range of path differences from zero to some maximum displacement $D$ and to apply the appropriate transform-a cosine transform in the case of a symmetric (AM) interferogram and a sine transform in the case of an antisymmetric (PM) interferogram. Note that the upper limits of the integrals in (4) and (5) will be replaced by the finite value $D$. Due to the difficulty of ensuring that sampling occurs at exactly ZPD, and also because of imperfections in the optical components, perfect symmetry (or antisymmetry) is rarely obtainable in practice [9]. For this reason it is more usual to record a "two-sided interferogram" over the range $-D-+D$ about the ZPD and to apply a full complex Fourier transform to the data. This will produce the result

$$
R(\bar{\nu})=P(\bar{\nu})+i Q(\bar{\nu})
$$

where $P(\bar{\nu})$ is the real (cosine) and $Q(\bar{\nu})$ the imaginary (sine) component. An antisymmetric PM interferogram should have $Q(\bar{\nu})$ as the dominant component and with perfect antisymmetry $P(\bar{\nu})$ should vanish completely. Use of the complex transform eliminates spectral distortion due to lack of alignment and other optical flaws as well as overcoming the ZPD offset problem mentioned above. The spectral intensity of the detected radiation is

$$
S(\bar{\nu})=\left[P^{2}(\bar{\nu})+Q^{2}(\bar{\nu})\right]^{1 / 2} .
$$

The function computed from a PM interferogram is actually of the form $B(\bar{\nu}) J_{1}(2 \pi \bar{\nu} a)$ where $B(\bar{\nu})$ is the true spectral intensity of the source as may be seen from (5). The Bessel function which arises as a side-effect of phase modulation will have a windowing effect on the profile of transmission spectra [4] but will not cause any problems as long as the amplitude of the modulation is sufficiently low to ensure that the maximum frequency of interest lies within the range determined by the first zero of $J_{1}$. Cutoff occurs at a wavenumber given by $\bar{\nu}_{c}=0.61 / a$ where $a$ is the amplitude of the modulation in terms of optical path difference. The presence of $J_{1}$ will not affect the profile of an absorption spectrum since this is calculated from the ratio of two transmission spectra and the Bessel functions will cancel, being equal in both cases.

\section{B. The Discrete Fourier Transform (DFT)}

For the purpose of calculating the Fourier transform of the sampled interferogram we replace the continuous integral by a discrete summation over $2 N$ values of sampled 
data. Thus

$$
R(\bar{\nu})=\int_{-D}^{D} b(x) \exp (2 \pi i \bar{\nu} x) d x
$$

becomes

$$
R\left(\bar{\nu}_{k}\right)=\Delta x \sum_{l=-N}^{N-1} F\left(x_{l}\right) \exp \left(2 \pi i \bar{\nu}_{k} x_{l}\right)
$$

or, more conveniently, from the properties of the DFT [7]

$$
R\left(\bar{\nu}_{k}\right)=\Delta x \sum_{l=-N}^{N-1} F\left(x_{l}\right) \exp \left(2 \pi i \bar{\nu}_{k} x_{l}\right)
$$

where $k$ and $l$ are integers lying in the range $0-2 N-1$. The variables $\Delta \vec{\nu}$ and $\Delta x$ represent, respectively, the spacing of the frequency abscissas (in wavenumbers) and the increment in optical path difference.

In general, programs which compute the DFT of a sequence of values employ a special algorithm-fast Fourier transform (FFT) which significantly reduces computation time. The summation of (10) is calculated without the scaling factor, $\Delta x$, which may be subsequently inserted by the user if required. We make use of an FFT program developed by Singleton [8]. A DFT operation performed on a set of $2 N$ real data points can be used to generate $2 N$ points in the frequency domain. However, only the first $N+1$ points yield useful information. The remaining points are complex conjugates of the first set corresponding to negative frequencies and have no physical significance. Thus if we have an interferogram with $2 N$ data points and a spatial sampling interval of $\Delta x$ corresponding to each increment of optical path difference, we can obtain spectral information at $N+1$ points. The sampling theorem [9] defines the maximum wavenumber at which we can obtain information as $\bar{\nu}_{\max }=1 / 2 \Delta x$. The range 0 to $\bar{\nu}_{\max }$ is subdivided into $N$ equal intervals and the spacing of abscissas in the frequency (wavenumber) domain is

$$
\Delta \bar{\nu}=\frac{1}{2 N \Delta x} .
$$

For our system $\Delta x$ is $5.0 \mu \mathrm{m}$ or twice the mirror displacement produced by a single motor step. Spectral information may be obtained up to $1000 \mathrm{~cm}^{-1}$. In practice, the useful spectral range is limited to a wavenumber below this value. The cutoff wavenumber will depend on the beam divider thickness [10], the material used in the windows of the cell holding the sample under investigation, and the frequency response characteristic of the detector.

\section{Apodization and Spectral Resolution}

The DFT is performed on a discrete set of values. An analysis of the effects of this truncation [10] shows that it produces unwanted subsidiary maxima accompanying real spectral features. The subsidiary maxima can be smoothed out by using a mathematical technique known as apodization which makes use of a windowing function to reduce the abruptness of truncation at the end points of the interferogram. Apodization results in a certain loss of spectral resolution but produces a smoother spectral profile. It can be shown [4] that the actual resolution available from an apodized interferogram is

$$
\Delta \bar{\nu}_{a}=0.9 / D
$$

where $D$ is the maximum optical path difference introduced in recording the interferogram. Since $D=N \Delta x$, it follows from (11) and (12) that $\Delta \bar{\nu}_{a}=2 \Delta \bar{\nu}$ where $\Delta \bar{\nu}$ is the actual spacing of spectral information produced by the DFT. Features in the apodized spectrum must be separated by at least twice this interval $\Delta \bar{\nu}$ if they are to be recognized as distinct. The overall improvement in the smoothness of the spectrum is generally considered to be adequate compensation for the effective halving of resolution which it produces. Increased resolution, where required, is generally obtained by increasing the range over which the interferogram is recorded rather than by dispensing with apodization.

Since the far infrared (FIR) absorption features of polar liquids which we study are broad band, a resolution of 4 $\mathrm{cm}^{-1}$ is usually adequate. For this resolution we require a 1000 point interferogram. Collection of 1000 data points corresponds to a mirror travel of $0.125 \mathrm{~cm}$, on either side of the ZPD position. This introduces a maximum optical path difference of $0.25 \mathrm{~cm}$. From (12) $\Delta \bar{\nu}_{a}=3.6 \mathrm{~cm}^{-1}$ which on rounding off gives a resolution of $4 \mathrm{~cm}^{-1}$.

\section{Necessity for Proper Band Limiting of the Detected Signal}

In studies of the FIR absorption spectrum of liquid acetonitrile, Evans [11] observed and reported fine spectral details particularly in the $30-120 \mathrm{~cm}^{-1}$ region. These observations were made using an experimental system similar to the one described in this paper. The observed features still remained after improvements had been made in the stability and accuracy of the system and seemed to be present in most liquids. The repeatability of these observations strongly suggested that the FIR absorption spectra of the liquids studied were not broad band and featureless as previously supposed. Some researchers in this area were convinced that a new phenomenon of local order in liquids had been observed [12].

In response to a paper on this subject reported at a conference, Genzel [13] proposed that the observed spectral details were not genuine and had their origins in the "aliasing" effect of the DFT. He suggested that absorption features at near infrared frequencies were being superimposed onto the FIR because the detected signal was not properly band limited. A formal treatment of the aliasing phenomenon based on discussions with Scaife [14] is presented here to show the necessity of using a filter to remove unwanted near infrared radiation.

We start by considering a continuous analog signal $b(x)$ and its spectrum $B(\bar{\nu})$ obtained by Fourier transformation

$$
B(\bar{\nu})=\int_{-\infty}^{\infty} b(x) \exp (2 \pi i \bar{\nu}) d x
$$




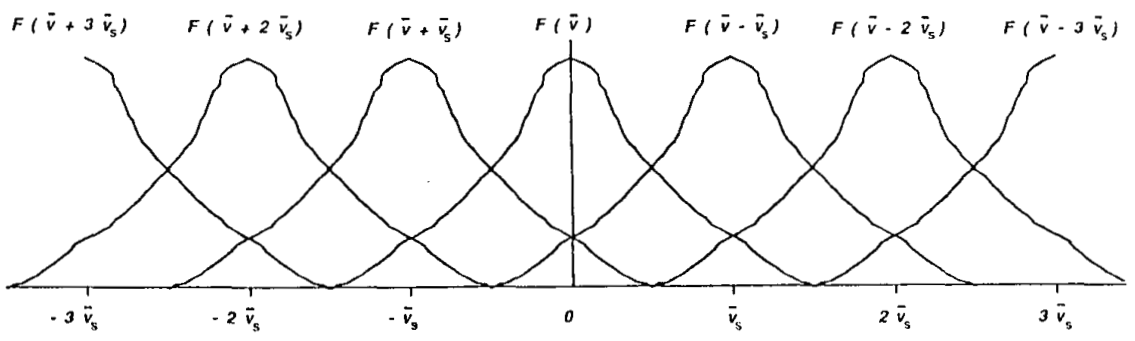

(a)

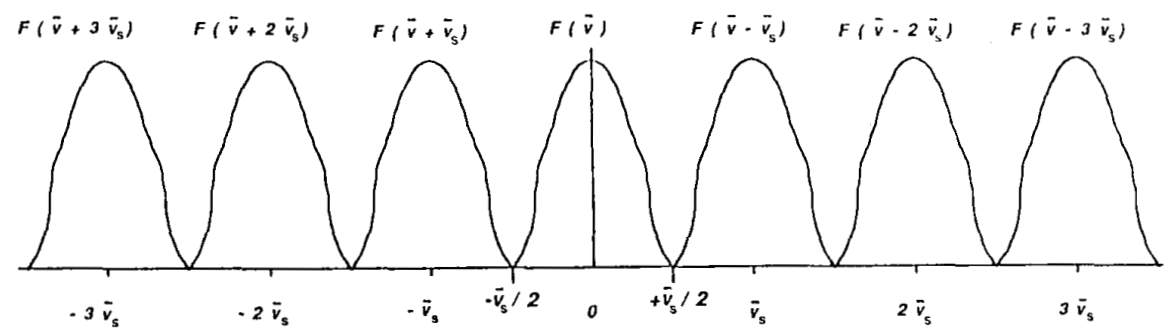

(b)

Fig. 4. The spectrum of a sampled signal is an infinite set of spectral aliases: (a) Signal not band limited-aliases overlap. (b) Band-limited signal-no alias overlap.

Here $b(x)$ corresponds to an infinite two-sided interferogram. If we sample $b(x)$ at intervals of $\Delta x$ we produce a new "digitized"' signal $b_{s}(x)$ which may be written as the product of the original analog signal and a sampling function which we represent as an infinite series of delta functions

$$
b_{s}(x)=\sum_{k=-\infty}^{\infty} b(x) \delta(x-k \Delta x)
$$

The sampling function is a periodic function of period $\Delta x$ and may be expressed as the Fourier series

$$
\sum_{k=-\infty}^{+\infty} \delta(x-k \Delta x)=\sum_{k=-\infty}^{+\infty} C_{k} \exp \left(\frac{-2 \pi i k x}{\Delta x}\right) .
$$

We can evaluate the coefficients of this expansion by integration [15] to give $C_{k}=1 / \Delta x$. Hence,

$$
b_{s}(x)=\frac{1}{\Delta x} \sum_{k=-\infty}^{+\infty} b(x) \exp \left(-\frac{2 \pi i k x}{\Delta x}\right) .
$$

We now turn our attention to $B_{s}(\vec{\nu})$, the spectrum of the sampled signal which we may evaluate by means of Fourier transformation as in (14)

$$
\begin{aligned}
& B_{s}(\bar{\nu})=\frac{1}{\Delta x} \int_{-\infty}^{+\infty} \sum_{k=-\infty}^{+\infty} b(x) \exp \left(-\frac{2 \pi i k x}{\Delta x}\right) \exp (2 \pi i \bar{\nu} x) \\
& B_{s}(\bar{\nu})=\frac{1}{\Delta x} \int_{-\infty}^{+\infty} \sum_{k=-\infty}^{+\infty} b(x) \exp \left[2 \pi i x\left(\bar{\nu}-k \bar{\nu}_{s}\right)\right] d x
\end{aligned}
$$

where we define a "sampling frequency" $\bar{\nu}_{s}=1 / \Delta x$. Interchanging the order of summation and integration gives

$$
\begin{aligned}
& B_{s}(\bar{\nu})=\frac{1}{\Delta x} \sum_{k=-\infty}^{+\infty} \int_{-\infty}^{\infty} b(x) \exp \left[2 \pi i x\left(\bar{\nu}-k \bar{\nu}_{s}\right)\right] d x \\
& B_{s}(\bar{\nu})=\frac{1}{\Delta x} \sum_{k=-\infty}^{+\infty} B\left(\bar{\nu}-k \bar{\nu}_{s}\right) .
\end{aligned}
$$

The spectrum of the sampled signal is actually an infinite series. Each term in the series is the spectrum of the original signal scaled by a factor of $1 / \Delta x$ and centered about an integral multiple of the sampling frequency, $\bar{\nu}_{s}$. The sampling process gives rise to an infinite series of spectral "aliases." If the spectrum of the analog signal extends beyond the critical value of $\bar{\nu}_{s} / 2$ then at any frequency the value of $B_{s}(\bar{\nu})$ will be the sum of contributions from all the spectral aliases. In such a case it will not be possible to recover the true spectrum of the analog signal by Fourier transformation of its digitized version. Spurious information arising from the overlap of the aliased spectra will be introduced into the calculated spectrum. To avoid this situation it is necessary that the signal to be sampled contains no spectral components at frequencies higher than $\bar{\nu}_{s} / 2$. This is essentially a restatement of the sampling theorem referred to previously. The above condition can be satisfied if a suitable filter is used to limit the bandwidth of the sampled signal. The phenomenon of aliasing is illustrated in Fig. 4. The upper diagram shows the overlap which arises when a spectrum is not bandlimited in accordance with the sampling theorem. The lower diagram shows the elimination of alias overlap by appropri- 


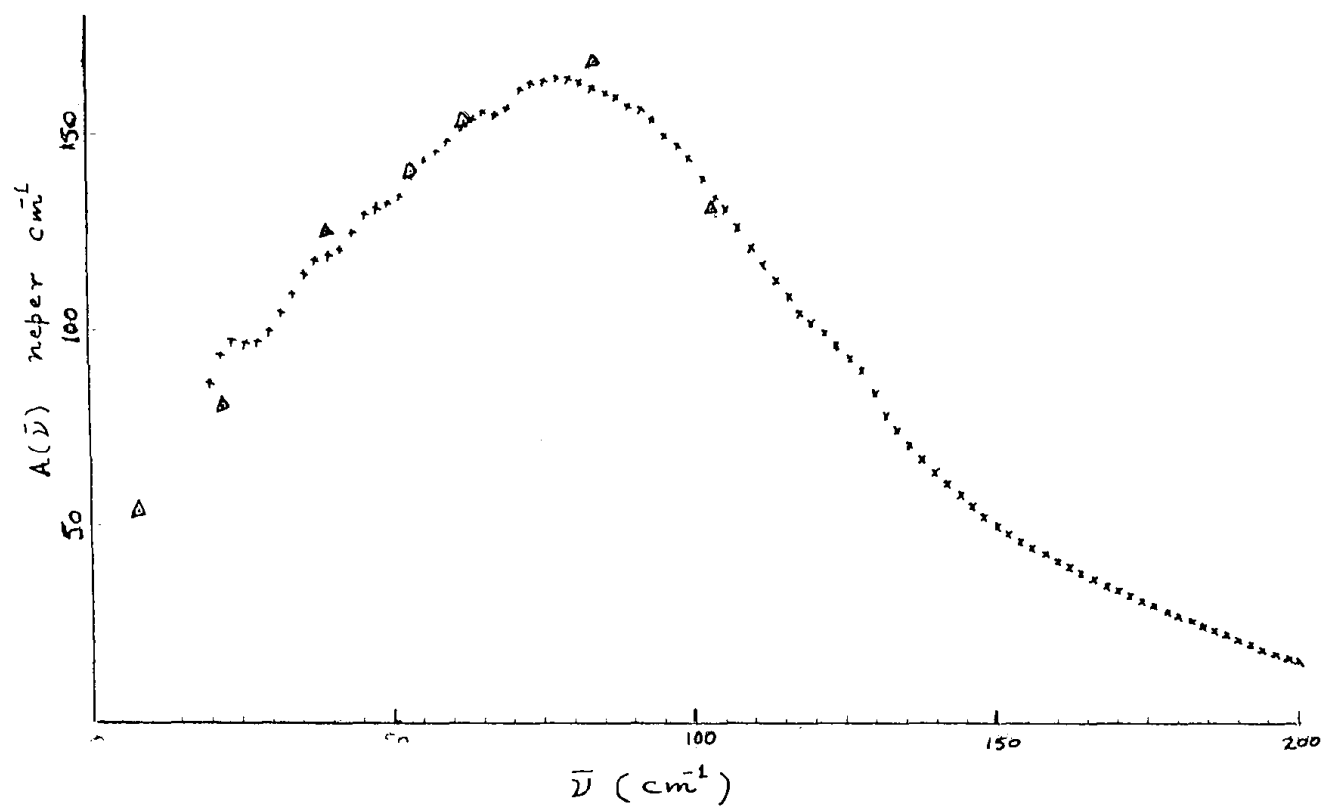

Fig. 5. Absorption spectra of dischloromethane using automated interferometric system. Laser points $\triangle$.

ate bandlimiting. In our system, we use a thin layer of black polyethylene in front of the detector window to exclude near infrared, and hence, avoid the problems associated with aliasing.

\section{Data Processing Software}

The processing of the interferometric data is carried out on a VAX 11/730 minicomputer using a Fortran 77 program written for this purpose. Each recorded interferogram is scanned to locate the midpoint or ZPD ordinate. $2 N$ data values are then selected about the midpoint. The value of $N$ depends on the resolution required and for $4-\mathrm{cm}^{-1}$ resolution $N=500$. The data is prepared for the FFT routine by sorting into two arrays $A$ and $B$ each of dimension $N$, with even-numbered ordinates stored in $A$ and odd-numbered ordinates stored in $B$. Half the mean value of the data is subtracted from each ordinate to obtain $b(x)$ as per (3). An apodizing function of the form $C^{*} \sin ^{2}(D * I)$ is applied to each ordinate where $C=$ $1 / 8 N, D=\pi / 2 N$, and $I$ is an indexing variable in the range $0 \cdots 2 N$. The program performs the subtraction and apodization operations as it is sorting the data. The $2 N$ data points are presented to the FFT routine as a set of $N$ complex values thereby making efficient use of a complex transform of dimension $N$. The FFT routine is called followed by a companion routine REALT which unscrambles the output of the FFT [8] and produces a sequence of values which is the complex transform of the original $2 N$ real data values. The power spectrum is then calculated at each wavenumber $\bar{\nu}$ as

$$
S(\bar{\nu})=\left[A^{2}(\bar{\nu})+B^{2}(\bar{\nu})\right]^{1 / 2} .
$$

Calculation of the absorption spectrum of a specimen in- volves computing the ratio of two transmission spectra recorded at different path lengths of the specimen. The Beer-Lambert law relates incident power, transmitted power, and specimen path length using the power absorption coefficient, $A(\nu)$ :

$$
S(\bar{\nu})=S_{0}(\bar{\nu}) \exp [-\mathbf{A}(\bar{\nu}) d]
$$

where $S(\bar{\nu})$ is the transmitted power, $S_{0}(\bar{\nu})$ the incident power, and $d$ the specimen thickness. If $S_{1}(\bar{\nu})$ is the spectrum recorded with an amplifier gain $G_{1}$ for a specimen thickness $d_{1}$ and $S_{2}(\bar{\nu})$ is the spectrum recorded with an amplifier gain $G_{2}$ for a specimen thickness $d_{2}$, we can write

$$
A(\bar{\nu})=\left(d_{2}-d_{1}\right)^{-1} \ln \left[\left(S_{1}(\bar{\nu}) / S_{2}(\bar{\nu})\right) * G\right]
$$

where $G=G_{2} / G_{1}$. The parameters used by the program to calculate absorption spectra i.e., the spectra to be ratioed, the relevant gain figures, and the path difference are read from a file set up by the user.

\section{EXPERIMENTAL Results}

The absorption spectra obtained using an FTS system may be compared with measurements made at discrete wavenumbers using a FIR laser. This serves as a useful check of the reliability of the interferometric system. We carried out measurements on two commonly studied polar liquids chloromethane $\left(\mathrm{CH}_{2} \mathrm{Cl}_{2}\right)$ and methyl iodide $\left(\mathrm{CH}_{3} \mathrm{I}\right)$ using our FTS system. A 100 gauge melinex beam splitter was used to cover the range $20-125 \mathrm{~cm}^{-1}$. For wavenumbers above $125 \mathrm{~cm}^{-1}$ a $12.5 \mu \mathrm{m}$ polyethylene beam splitter was used. The absorption spectrum of $\mathrm{CH}_{2} \mathrm{Cl}_{2}$ is shown in Fig. 5 and some values of the absorption coefficient which have been measured recently 
[16] using a tunable FIR molecular laser system are superimposed on the interferometric spectrum. The laser system [17] produces coherent submillimeter radiation by optical pumping of methanol or methyl iodide vapor using a $\mathrm{CO}_{2}$ laser as a primary power source. We have found that there is agreement between the two experimental methods to within about 2 percent.

\section{ConClusions}

An NPL-Grubb-Parsons modular cube interferometer has been automated to produce absorption spectra of liquids in the wavenumber range $10-400 \mathrm{~cm}^{-1}$. The optical system of the interferometer is very simple, using only one TPX lens in front of the detector to focus the broadband submillimeter radiation. More sophisticated commercial interferometers usually include more elaborate optical systems for collimating and focusing the radiation. Although our system lacks the complexity of such apparatus, it can produce results which compare favorably with those from a submillimeter laser system. The advantage of an interferometer over a laser for spectroscopic work is that it can rapidly produce a high resolution spectrum over a wide wavenumber range once the necessary interferometric data is available. Working with a $4-\mathrm{cm}^{-1}$ resolution on our system, it requires about $35 \mathrm{~min}$ to collect the data for each transmission spectrum. A laser can only be used to make measurements at discrete wavenumbers. To be of use in spectroscopic work it should be tunable over a wide spectral range. Laser systems are of great importance in the study of liquids which absorb heavily in the FIR (e.g., water) [17]. In the case of heavily absorbing liquids the relative weakness of broad-band sources necessitates the use of very thin specimen path lengths. The difficulty of accurately estimating the path length makes the calculated values of the absorption index rather unreliable. Laser systems can produce results su- perior to those obtainable with conventional FTS in such cases.

\section{ACKNOWLEDGMENT}

The authors would like to thank S. White and P. Gorman for their help. Drs. G. Evans, U.M.S. Murthy, and J. Birch are thanked for useful discussions.

\section{REFERENCES}

[1] H. A. Gebbie and G. A. Vanasse, "Interferometric spectroscopy in the far infra-red," Nature, vol. 178, p. 432, Aug 1956.

[2] J. Strong and G. A. Vanasse, "Interferometric spectroscopy in the far infrared," J. Opt. Soc. Amer., vol. 49, pp. 844-850, 1959.

[3] F. A. Jenkins and H. E. White, Fundamentals of Optics. New York: McGraw-Hill, 1957, p. 206.

[4] J. Chamberlain, The Principles of Interferometric Spectroscopy. New York: Wiley-Interscience, 1979

[5] P. Horowitz and W. Hill, The Art of Electronics. Cambridge, MA: Cambridge Univ. Press, 1985.

[6] RS Data Sheet 4816, RS Components Ltd, London, U.K.

[7] E. O. Bringham, The Fast Fourier Transform. Englewood Cliffs, New Jersey: Prentice-Hall Inc., 1974.

[8] R. C. Singleton, "An algorithm for calculating the mixed-radix Fourier transform,"'IEEE Trans. Audio Electroacous., vol. AU-17, pp. 103-113, June 1969.

[9] S. A. Tretter, Introduction to Discrete Time Signal Processing. New York: Wiley, 1976.

[10] G. W. Chantry, Submillimetre Spectroscopy. London, U.K.: Academic, 1971.

[11] G. J. Evans, "Spectral detail in liquid acetonitrile at far infrared frequencies,"' Chem. Phys. Lett., vol. 99, pp. 173-176, July 1983.

[12] M. W. Evans, S. J. Abas, G. J. Evans, and C. J. Reid, "Far infrared peaks in molecular liquids," Physica Scripta, vol. 32, pp. 236-247, 1985.

[13] L. Genzel, "Dielectrics society meeting," Univ. College of North Wales, Bangor, U.K., 1985.

[14] B. K. P. Scaife, private communication.

[15] E. Kreysig, Advanced Engineering Mathematics. New York: Wiley, 1979.

[16] J. K. Vij and F. Hufnagel, "Millimeter and submillimeter wave laser spectrometer for liquids," to be published.

[17] J. K. Vij, F. Hufnagel, M. Helker, and C. J. Reid, "Some new FIR laser lines of optically pumped $\mathrm{CH}_{3} \mathrm{OH}, \mathrm{CH}_{3} \mathrm{OD}, \mathrm{CH}_{3} \mathrm{I}, \mathrm{CH}_{3} \mathrm{Br}$ and $\mathrm{CD}_{2} \mathrm{Cl}_{2}$," IEEE J. Quantum Electron., vol. QE-22, pp. 1123-1129, July 1986 . 